\title{
ALGEBRAIC THEORIES OF BRACKETS AND RELATED (CO)HOMOLOGIES
}

\author{
I. Krasil'shchik
}

\begin{abstract}
A general theory of the Frölicher-Nijenhuis and Schouten-Nijenhuis brackets in the category of modules over a commutative algebra is described. Some related structures and (co)homology invariants are discussed, as well as applications to geometry.
\end{abstract}

Keywords Frölicher-Nijenhuis bracket · Schouten-Nijenhuis bracket · Poisson structures · Integrability $\cdot$ Nonlinear differential equations $\cdot$ Hamiltonian formalism $\cdot$ Algebraic approach

PACS 02.10.Hh $\cdot$ 02.30.Jr $\cdot 02.30 . \mathrm{Ik}$

Mathematics Subject Classification (2000) $58 \mathrm{~J} 10 \cdot 58 \mathrm{H} 15 \cdot 37 \mathrm{~K} 10$

\section{Introduction}

Bracket structures play an important role in classical differential geometry (see, for example, Refs. [3,4, 19, 20,21, 22, 23]), Poisson geometry (e.g., [17]), and the theory of integrable systems (Refs. [5,6,9]). Being initially of a geometrical nature, these brackets found exact counterparts in abstract algebra, in the framework of Vinogradov's theory of algebraic differential operators, [25] (see also book [15]). It became clear that many geometrical constructions (such as the ones we meet in Hamiltonian mechanics or in partial differential equations; cf. with Refs. [27] and [7], respectively) may be more or less exactly expressed using the language of commutative algebra.

In this paper, I collected together my old results on the algebraic theory of the FrölicherNijenhuis and Schouten-Nijenhuis brackets and related homological and cohomological theories (for shortness, I call these brackets Nijenhuis and Schouten ones). These results were initially published in papers [7, 8, 9, 13]. The results exposed below are easily generalized to the case of super-commutative algebras (see [10]) and, being slightly modified, can be incorporated in Lychagin's "colored calculus" (see Ref.[16]).

This work was supported in part by the NWO-RFBR grant 047.017.015, RFBR-Consortium E.I.N.S.T.E.I.N. grant 06-01-92060 and RFBR-CNRS grant 08-07-92496.

Iosif Krasil's shchik

Independent University of Moscow, B. Vlasevsky 11, 119002 Moscow, Russia

E-mail: josephk@diffiety.ac.ru 
To simplify exposition, I shall always assume that the algebra $A$ is such that the module $\Lambda^{1}(A)$ (see Sec. 3.2) of 1 -forms is projective and of finite type.

\section{A general scheme}

This scheme was first presented in Ref. [26].

Let $\mathbb{k}$ be a field, char $\mathbb{k} \neq 2$. Let also $\mathbb{P}=\sum_{k \in \mathbb{Z}} \mathbb{P}_{k}, \mathbb{Q}=\sum_{k \in \mathbb{Z}} \mathbb{Q}_{k}$ be graded vector spaces and $\mathbb{Q}$ be endowed with a differential

$$
d: \mathbb{Q}_{k} \rightarrow \mathbb{Q}_{k+1}, \quad d^{2}=0 .
$$

Assume that there exists a graded monomorphism

$$
\varphi: \mathbb{P} \rightarrow \operatorname{Hom}_{\mathbb{k}}^{\mathrm{gr}}(\mathbb{Q}, \mathbb{Q}), \quad \varphi\left(\mathbb{P}_{\alpha}\right) \subset \operatorname{Hom}_{\mathbb{k}}^{\alpha+\beta}(\mathbb{Q}, \mathbb{Q}), \quad \beta=\operatorname{gr} \varphi,
$$

and define the "Lie derivative"

$$
\mathrm{L}_{p}^{\varphi}=[d, \varphi(p)], \quad p \in \mathbb{P} .
$$

Here and everywhere below $[\cdot, \cdot]$ denotes the graded commutator. If we are lucky then for two elements $p \in \mathbb{P}_{\alpha}, p^{\prime} \in \mathbb{P}_{\alpha^{\prime}}$ we can define their $\varphi$-bracket by

$$
\left.\mathrm{L}_{\llbracket p, p^{\prime} \rrbracket}^{\varphi}=\left[\mathrm{L}_{p}^{\varphi}, \mathrm{L}_{p^{\prime}}^{\varphi}\right], \quad \llbracket\left[p, p^{\prime}\right]\right] \in \mathbb{P}_{\alpha+\alpha^{\prime}+\beta+1} .
$$

In some interesting cases we are lucky indeed.

\section{Algebraic calculus}

Let us introduce the basic notions of the calculus over commutative algebras that will be needed below (see Refs. [12, 15, 25] for details).

\subsection{Differential operators}

Consider a unitary commutative associative $\mathbb{k}$-algebra $A$ and $A$-modules $P$ and $Q$.

Definition $1 \mathrm{~A} \mathbb{k}$-linear map $\Delta: P \rightarrow Q$ is a differential operator (DO) of order $\leq k$ if

$$
\left[a_{0},\left[a_{1},\left[\ldots\left[a_{k}, \Delta\right] \ldots\right]\right]\right]=0
$$

for all $a_{0}, \ldots, a_{k} \in A$.

The set of all DOs $P \rightarrow Q$ forms an $A$-bimodule denoted by $\operatorname{Diff}_{*}(P, Q)$. An operator $X: A \rightarrow$ $P$ is called a $P$-valued derivation if

$$
X(a b)=a X(b)+b X(a), \quad a, b \in A .
$$

The module of these derivations is denoted by $D_{1}(P)$. Define by induction the modules

$$
D_{i}(P)=\left\{X \in D_{1}\left(D_{i-1}(P)\right) \mid X(a, b)+X(b, a)=0\right\}, \quad i \geq 2,
$$

and set formally $D_{0}(P)=P$. Elements of $D_{i}(P)$ are called multiderivations.

Remark 1 Let $M$ be a smooth manifold and $A=C^{\infty}(M)$. Let also $\pi$ and $\xi$ be vector bundles over $M$ and $P=\Gamma(\pi), Q=\Gamma(\xi)$ be the modules of their smooth sections. Then Definition 1 gives the classical notion of a linear differential operator. 


\subsection{Differential forms}

Proposition 1 The correspondence $P \Rightarrow D_{i}(P)$ is a representable functor from the category of A-modules to itself. The corresponding representative object is denoted by $\Lambda^{i}=\Lambda^{i}(A)$ and called the module of differential $i$-forms of the algebra A. In particular, there exists a natural derivation $d: A \rightarrow \Lambda^{1}$ such that any derivation $X \in D_{1}(P)$ uniquely decomposes as $X=\psi_{X} \circ d$, where $\psi_{X} \in \operatorname{Hom}_{A}\left(\Lambda^{1}, P\right)$. The module $\Lambda^{1}$ is generated by the elements of the form $d a, a \in A$, while $\Lambda^{i}$ are exterior powers of $\Lambda^{1}$. This leads to the complex

$$
0 \longrightarrow A \stackrel{d}{\longrightarrow} \Lambda^{1} \longrightarrow \cdots \longrightarrow \Lambda^{i} \stackrel{d}{\longrightarrow} \Lambda^{i+1} \longrightarrow \cdots
$$

called the de Rham complex of $A$.

\subsection{Exterior products}

Due to the above formulated proposition, the module $\Lambda^{*}=\sum_{i} \Lambda^{i}$ is a Grassmannian algebra with the exterior, or wedge product

$$
\wedge: \Lambda^{i} \otimes_{A} \Lambda^{j} \rightarrow \Lambda^{i+j}
$$

A similar operation

$$
\wedge: D_{i}(A) \otimes_{A} D_{j}(P) \rightarrow D_{i+j}(P)
$$

is introduced by induction in $D_{*}(P)=\sum_{i} D_{i}(P)$. Namely, for $i+j=0$ we set

$$
a \wedge p=a p, \quad a \in D_{0}(A)=A, \quad p \in D_{0}(P)=P,
$$

and

$$
(X \wedge Y)(a)=X \wedge Y(a)+(-1)^{j} X(a) \wedge Y, \quad i+j>0,
$$

$X \in D_{i}(A), Y \in D_{j}(P)$. In this way, $D_{*}(A)$ becomes a Grassmannian algebra, $D_{*}(P)$ being a module over $D_{*}(A)$.

\subsection{Inner product}

The inner product

$$
\mathrm{i}: D_{i}(P) \otimes_{A} \Lambda^{j} \rightarrow \begin{cases}P \otimes_{A} \Lambda^{j-i}, & j \geq i \\ D_{i-j}(P), & j \leq i\end{cases}
$$

is defined by induction. If $i=0$ we set

$$
\mathrm{i}(p \otimes \omega)=p \otimes \omega, \quad p \in P=D_{0}(P), \quad \omega \in \Lambda^{j},
$$

and for $j=0$ we set

$$
\mathrm{i}(X \otimes a)=a X, \quad a \in A=\Lambda^{0}, \quad X \in D_{i}(P) .
$$

If $i, j>0$ we set

$$
\mathrm{i}(X \otimes d a \wedge \omega)=\mathrm{i}(X(a) \otimes \omega) .
$$

We shall use the notation

$$
\mathrm{i}_{X} \omega=\left\{\begin{array}{ll}
\mathrm{i}(X \otimes \omega), & i \geq j, \\
0, & i<j,
\end{array} \quad \text { and } \quad \mathrm{i}_{\omega} X= \begin{cases}\mathrm{i}(X \otimes \omega), & i \leq j, \\
0, & i>j\end{cases}\right.
$$


Remark 2 When $P=\Lambda^{k}$ and $j \geq i$, the inner product may be completed to the following operation

$$
D_{i}\left(\Lambda^{k}\right) \otimes_{A} \Lambda^{j} \stackrel{\mathrm{i}}{\longrightarrow} \Lambda^{k} \otimes_{A} \Lambda^{j-i} \stackrel{\wedge}{\longrightarrow} \Lambda^{k+j-i}
$$

which will be also called the inner product.

Remark 3 Let $X \in D_{i}(P)$ and $\omega \in \Lambda^{j}$. Then the maps

$$
\mathrm{i}_{X}: \Lambda^{*} \rightarrow P \otimes_{A} \Lambda^{*}
$$

and

$$
\mathrm{i}_{\omega}: D_{*}(P) \rightarrow D_{*}(P)
$$

are super-differential operators of order $i$ and $j$, respectfully.

\section{The Schouten bracket}

We define here the Schouten bracket, describe its properties and related (co)homologies. Some applications are also discussed.

4.1 Definition and existence

Let $X \in D_{i}(A)$. Consider the Lie derivative

$$
\mathrm{L}_{X}=d \circ \mathrm{i}_{X}-(-1)^{i} \dot{\mathrm{i}}_{X} \circ d=\left[d, \mathrm{i}_{X}\right]: \Lambda^{j} \rightarrow \Lambda^{j-i} .
$$

Theorem 1 For any two elements $X \in D_{i}(A)$ and $X^{\prime} \in D_{i^{\prime}}(A)$ there exists a uniquely defined element $\left[\left[X, X^{\prime}\right]^{\mathrm{s}} \in D_{i+i^{\prime}-1}(A)\right.$ such that

$$
\left[\mathrm{L}_{X}, \mathrm{~L}_{X^{\prime}}\right]=\mathrm{L}_{\left[X, X^{\prime}\right]^{\mathrm{s}}} .
$$

This element is called the Schouten bracket of $X$ and $X^{\prime}$.

Proof We establish existence of $\left[\left[X, X^{\prime}\right]\right]^{\mathrm{s}}$ by induction. For $i^{\prime}=0$ we set

$$
[[X, a]]^{\mathrm{s}}=X(a), \quad a \in A=D_{0}(A),
$$

and similarly for $i=0$

$$
\left[\left[a, X^{\prime}\right]\right]^{\mathrm{s}}=(-1)^{i^{\prime}} X^{\prime}(a)
$$

If $i, i^{\prime}>0$ we set

$$
\left[\left[X, X^{\prime}\right]\right]^{\mathrm{s}}(a)=\left[\left[X, X^{\prime}(a)\right]^{\mathrm{s}}+(-1)^{i^{\prime}-1}\left[\left[X(a), X^{\prime}\right]\right]^{\mathrm{s}} .\right.
$$

It is easily checked that thus defined bracket enjoys the needed property. 


\subsection{Properties}

Proposition 2 Let $X, X^{\prime}, X^{\prime \prime} \in D_{*}(A)$ be multiderivations of degree $i, i^{\prime}$ and $i^{\prime \prime}$ respectively. Then:

1. $\left[\left[X, X^{\prime}\right]\right]^{\mathrm{s}}+(-1)^{(i-1)\left(i^{\prime}-1\right)}\left[\left[X^{\prime}, X\right]\right]^{\mathrm{s}}=0$,

2. $\left.\left[\left[X,\left[\left[X^{\prime}, X^{\prime \prime}\right]\right]^{\mathrm{s}}\right]\right]^{\mathrm{s}}=\left[\left[\left[X, X^{\prime}\right]\right]^{\mathrm{s}}, X^{\prime \prime}\right]\right]^{\mathrm{s}}+(-1)^{(i-1)\left(i^{\prime}-1\right)}\left[\left[X^{\prime},\left[\left[X, X^{\prime \prime}\right]\right]^{\mathrm{s}}\right]\right]^{\mathrm{s}}$,

3. $\left[\left[X, X^{\prime} \wedge X^{\prime \prime}\right]\right]^{\mathrm{s}}=\left[\left[X, X^{\prime}\right]\right]^{\mathrm{s}} \wedge X^{\prime \prime}+(-1)^{(i-1) i^{\prime}} X^{\prime} \wedge\left[\left[X, X^{\prime \prime}\right]\right]^{\mathrm{s}}$,

4. $\left[\left[X, X^{\prime}\right]\right]^{\mathrm{s}}=\left[X, X^{\prime}\right]$, if $i=i^{\prime}=1$,

5. $\mathrm{i}_{\left.\left[X, X^{\prime}\right]\right]^{\mathrm{s}}}=\left[\mathrm{L}_{X}, \mathrm{i}_{X^{\prime}}\right]$.

\section{Poisson structures}

To any bivector $\mathscr{P} \in D_{2}(A)$, one can put into correspondence a skew-symmetric bracket $\{a, b\}_{\mathscr{P}}=\mathscr{P}(a, b), a, b \in A$.

Proposition 3 The following statements are equivalent:

1. $\{a, b\}_{\mathscr{P}}$ satisfies the Jacobi identity;

2. $[[\mathscr{P}, \mathscr{P}]]^{\mathrm{s}}=0$;

3. $\partial_{\mathscr{P}} \circ \partial_{\mathscr{P}}=0$, where $\partial_{\mathscr{P}}=[[\mathscr{P}, \cdot]]^{\mathrm{s}}$.

Definition 2 If one of the previous conditions fulfills then:

1. $\mathscr{P}$ is called a Poisson structure and a pair $(A, \mathscr{P})$ is a Poisson algebra.

2. $\{\cdot, \cdot\}_{\mathscr{P}}$ is the Poisson bracket associated with $\mathscr{P}$.

3. $\mathscr{P}(a) \in D_{1}(A)$ are Hamiltonian derivations.

4. Derivations $X$ satisfying $X\{a, b\}_{\mathscr{P}}=\{X a, b\}_{\mathscr{P}}+\{a, X b\}_{\mathscr{P}}$ are canonical derivations.

\subsection{Example: algebraic $T^{*}$ (see Ref. [27])}

Let $\operatorname{Diff}_{*}(A)=\cup_{k \geq 0} \operatorname{Diff}_{k}(A)$ denote the algebra of all DOs $A \rightarrow A$. For any $\Delta \in \operatorname{Diff}_{k}(A)$ the coset $[\Delta]_{k}=\Delta \bmod \operatorname{Diff}_{k-1}(A)$ is called its symbol.

If $s_{1}=\left[\Delta_{1}\right]_{k_{1}}, s_{2}=\left[\Delta_{1}\right]_{k_{2}}$ are two symbols we define their product by

$$
s_{1} \cdot s_{2}=\left[\Delta_{1} \circ \Delta_{2}\right]_{k_{1}+k_{2}}
$$

and their bracket by

$$
\left\{s_{1}, s_{2}\right\}=\left[\Delta_{1} \circ \Delta_{2}-\Delta_{2} \circ \Delta_{1}\right]_{k_{1}+k_{2}-1} .
$$

In such a way we obtain the algebra of symbols

$$
S_{*}(A)=\sum_{k} \frac{\operatorname{Diff}_{k}(A)}{\operatorname{Diff}_{k-1}(A)} .
$$

Proposition 4 The above introduced algebra of symbols $S_{*}(A)$ is a graded commutative algebra with a graded Poisson bracket $\{\cdot, \cdot\}$. In the case $A=C^{\infty}(M)$ it coincides with the algebra of smooth functions on $T^{*} M$ polynomial along the fibers, while the bracket is the one defined by the canonical symplectic form $\Omega=d p \wedge d q$. 
Remark 4 The parallel between geometrical constructions and the corresponding algebraic modules is even deeper, though perhaps not so straightforward. As an example, let us describe how the canonical form $\rho=p d q$ is defined within the model under consideration (other illustrations can be found, e.g., in Refs. [11],14]).

Note first that exactly in the same way as it was done above one can define symbols of arbitrary operators $\Delta \in \operatorname{Diff}_{*}(P, Q)$. Moreover, under the assumption of Sec. 1 one has the isomorphism

$$
S_{*}(P, Q)=S_{*}(A) \otimes_{A} \operatorname{Hom}_{A}(P, Q) .
$$

Now, to define a 1-form, one needs to evaluate all derivations on this form. Let $X: S_{*}(A) \rightarrow R$ be such a derivation, $R$ being an $S_{*}(A)$-module. Since $A=S_{0}(A) \subset S_{*}(A)$, one can consider the restriction $\bar{X}=\left.\bar{X}\right|_{A}: A \rightarrow R$. Due to Eq. (1), one has

$$
[\bar{X}] \in S_{*}(A, R)=S_{*}(A) \otimes_{A} R,
$$

and we set

$$
\mathrm{i}_{\bar{X}} \rho=\mu_{S}(\bar{X}),
$$

where $\mu_{S}: S_{*}(A) \otimes_{A} R \rightarrow R$ is the multiplication. Consequently, we can define the form $\Omega=$ $d \rho$, but for general algebras it may be degenerate, contrary to the geometric case.

Remark 5 It may be also appropriate to discuss another parallel here. Namely, in geometry, 1-forms are identified with sections of the cotangent bundle. In algebra, the notion of section transforms to a homomorphism $\varphi: S_{*}(A) \rightarrow A=S_{0}(A)$ such that $\left.\varphi\right|_{A}=$ id. Consider a 1form $\omega \in \Lambda^{1}(A)$ and let us define the corresponding "section" $\varphi_{\omega}: S_{*}(A) \rightarrow A$. To this end, recall that in the case when $\Lambda^{1}(A)$ is a finite type projective module the algebra $\operatorname{Diff}_{*}(A)$ is generated by $A=\operatorname{Diff}_{0}(A)$ and $D_{1}(A)$. Therefore, any element $s \in S_{k}(A)$ is of the form $s=\sum\left[X_{\alpha_{1}}\right] \cdot \ldots \cdot\left[X_{\alpha_{k}}\right]$, where $X_{\alpha}$ are derivations. Then we set

$$
\varphi_{\omega}(s)=\sum \mathrm{i}_{X_{\alpha_{1}}}(\omega) \cdot \ldots \cdot \mathrm{i}_{X_{\alpha_{k}}}(\omega) .
$$

Conversely, let $\varphi: S_{*}(A) \rightarrow A$ be a homomorphism. To define the corresponding 1-form $\omega_{\varphi}$, we need to evaluate an arbitrary derivation $X: A \rightarrow P$ at it, where $P$ is an $A$-module. But

$$
[X] \in S_{*}(A, P)=S_{*}(A) \otimes_{A} P,
$$

and we set

$$
\mathrm{i}_{X}\left(\omega_{\varphi}\right)=\mu_{A}\left(\varphi \otimes \operatorname{id}_{P}([X])\right),
$$

where $\mu_{A}: A \otimes_{\mathbb{R}} P \rightarrow P$ is the multiplication.

\subsection{Poisson cohomologies}

Let $(A, \mathscr{P})$ be a Poisson algebra. The sequence

$$
0 \longrightarrow A \stackrel{\partial_{\mathscr{P}}}{\longrightarrow} D_{1}(A) \longrightarrow \cdots \longrightarrow D_{i}(P) \stackrel{\partial_{\mathscr{P}}}{\longrightarrow} D_{i+1}(P) \longrightarrow
$$

where $\partial_{\mathscr{P}}=[[\mathscr{P}, \cdot]]^{\mathrm{s}}$, is the Poisson complex of $A$ and its cohomologies $H^{i}(A, \mathscr{P})$ are the Poisson cohomologies.

Proposition 5 1. $H^{0}(A ; \mathscr{P})$ consists of Casimirs of $\mathscr{P}$ and coincides with the Poisson center of $A$. 
2. $H^{1}(A ; \mathscr{P})=\operatorname{Can}(\mathscr{P}) / \operatorname{Ham}(\mathscr{P})$. where $\operatorname{Can}(\mathscr{P})$ is the space of canonical derivations and $\operatorname{Ham}(\mathscr{P})$ consists of the Hamiltonian ones.

3. $H^{2}(A ; \mathscr{P})$ coincides with the set of classes of nontrivial infinitesimal deformations of the Poisson structure $\mathscr{P}$.

4. $H^{3}(A ; \mathscr{P})$ contains obstructions to prolongation of infinitesimal deformations up to formal ones.

5.3 Poisson homologies (see Ref. [2])

Take a Poisson algebra $(A, \mathscr{P})$ and consider the operator

$$
d_{\mathscr{P}}=\mathrm{L}_{\mathscr{P}}=\left[d, \mathrm{i}_{\mathscr{P}}\right]: \Lambda^{j} \rightarrow \Lambda^{j-1} .
$$

By definition of the Poisson structure, one has

$$
2 d_{\mathscr{P}} \circ d_{\mathscr{P}}=\left[d_{\mathscr{P}}, d_{\mathscr{P}}\right]=\left[\mathrm{L}_{\mathscr{P}}, \mathrm{L}_{\mathscr{P}}\right]=\mathrm{L}_{[\mathscr{P}, \mathscr{P}]]^{\mathrm{s}}}=0
$$

and one gets the complex

$$
\cdots \longrightarrow \Lambda^{j} \stackrel{d_{\mathscr{P}}}{\longrightarrow} \Lambda^{j-1} \longrightarrow \cdots \longrightarrow \Lambda^{1} \stackrel{d_{\mathscr{P}}}{\longrightarrow} A \longrightarrow
$$

whose homologies $H_{j}(A, \mathscr{P})$ are called the Poisson homologies of $(A, \mathscr{P})$. The action of $d_{\mathscr{P}}$ is fully defined by the following two properties:

$$
d_{\mathscr{P}}\left(\omega \wedge \omega^{\prime}\right)=\left(d_{\mathscr{P}} \omega\right) \wedge \omega^{\prime}+(-1)^{j} \omega \wedge d_{\mathscr{P}} \omega^{\prime}
$$

and

$$
d_{\mathscr{P}}(a d b)=\{a, b\}_{\mathscr{P}}, \quad a, b \in A .
$$

5.4 Hamiltonian filtrations

Let $\mathscr{H}^{1} \subset D_{*}(A)$ be the ideal generated by Hamiltonian derivations. Let

$$
\mathscr{H}^{p}=\underbrace{\mathscr{H}^{1} \wedge \cdots \wedge \mathscr{H}^{1}}_{p \text { times }}
$$

be its powers. Then

$$
D_{*}(A)=\mathscr{H}^{0} \supset \mathscr{H}^{1} \supset \ldots \supset \mathscr{H}^{p} \supset \mathscr{H}^{p+1} \supset \ldots
$$

is a filtration that generates a spectral sequence for Poisson cohomologies. In a dual way, the filtration

$$
0 \subset \mathscr{H}_{1} \subset \cdots \subset \mathscr{H}_{p} \subset \mathscr{H}_{p+1} \subset \ldots
$$

where

$$
\mathscr{H}_{p}=\left\{\omega \in \Lambda^{*} \mid \mathrm{i}_{X_{1}}\left(\ldots\left(\mathrm{i}_{X_{p}}(\omega)\right) \ldots\right)=0 \forall X_{i} \in \operatorname{Ham}(\mathscr{P})\right\}
$$

gives rise to a spectral sequence for Poisson homologies. 


\section{Extended Poisson bracket}

The Poisson bracket defined by a Poissonian bi-vector $\mathscr{P}$ can be extended to a super-bracket on the Grassmannian algebra $\Lambda^{*}(A)$.

Consider the differential $\partial_{\mathscr{P}}: D_{i}(A) \rightarrow D_{i+1}(A)$ and a form $\omega \in \Lambda^{j}$. Then we have the map

$$
\mathrm{L}_{\omega}^{\mathscr{P}}=\left[\partial_{\mathscr{P}}, \mathrm{i}_{\omega}\right]: D_{i}(A) \rightarrow D_{i-j+1}(A)
$$

Proposition 6 For any two forms $\omega \in \Lambda^{j}, \omega^{\prime} \in \Lambda^{j^{\prime}}$ the equality

$$
\mathrm{i}_{\left\{\omega, \omega^{\prime}\right\}_{\mathscr{P}}}=\left[\mathrm{L}_{\omega}^{\mathscr{P}}, \mathrm{i}_{\omega^{\prime}}\right]
$$

uniquely determines a form $\left\{\omega, \omega^{\prime}\right\} \mathscr{P} \in \Lambda^{j+j^{\prime}-1}$, which is called their Poisson bracket.

Proposition 7 The Poisson bracket of forms enjoys the following properties:

1. $\{a, d b\}_{\mathscr{P}}=-\{a, b\}_{\mathscr{P}}$,

2. $\{d a, d b\}_{\mathscr{P}}=d\{a, b\}_{\mathscr{P}}$,

3. $\left\{\omega, \omega^{\prime} \wedge \omega^{\prime \prime}\right\}_{\mathscr{P}}=\left\{\omega, \omega^{\prime}\right\} \mathscr{P} \wedge \omega^{\prime \prime}+(-1)^{(j-1) j^{\prime}} \omega^{\prime} \wedge\left\{\omega, \omega^{\prime \prime}\right\} \mathscr{P}$,

4. $\left\{\omega,\left\{\omega^{\prime}, \omega^{\prime \prime}\right\}_{\mathscr{P}}\right\}_{\mathscr{P}}=\left\{\left\{\omega, \omega^{\prime}\right\} \mathscr{P}, \omega^{\prime \prime}\right\} \mathscr{P}+(-1)^{(j-1)\left(j^{\prime}-1\right)}\left\{\omega^{\prime},\left\{\omega, \omega^{\prime \prime}\right\} \mathscr{P}\right\} \mathscr{P}$,

5. $\left\{\omega, \omega^{\prime}\right\} \mathscr{P}=-(-1)^{(j-1)\left(j^{\prime}-1\right)}\left\{\omega^{\prime}, \omega\right\} \mathscr{P}$,

6. $\mathrm{L}_{\left\{\omega, \omega^{\prime}\right\} \mathscr{P}}=\left[\mathrm{L}_{\omega}, \mathrm{L}_{\omega^{\prime}}\right]$.

Note that the first three properties may be taken for the constructive definition of the extended bracket.

\section{Commuting structures}

Two Poisson structures $\mathscr{P}$ and $\mathscr{P}^{\prime}$ commute, or are compatible if $\left[\left[\mathscr{P}, \mathscr{P}^{\prime}\right]^{\mathrm{s}}=0\right.$. This is equivalent to

$$
\partial_{\mathscr{P}} \circ \partial_{\mathscr{P} \prime}=0
$$

or to the fact that $\mu \mathscr{P}+\mu^{\prime} \mathscr{P}^{\prime}$ (the Poisson pencil) is a Poisson structure for all $\mu, \mu^{\prime} \in \mathbb{k}$.

The Magri scheme (see Ref [18]) that establishes existence of infinite series of commuting conservation laws for bi-Hamiltonian systems has an exact algebraic counterpart:

Theorem 2 Let $A$ be an algebra with two commuting Poisson structures $\mathscr{P}$ and $\mathscr{P}$ ' and assume that $H^{1}\left(A ; \mathscr{P}^{\prime}\right)=0$. Assume also that two elements $a_{1}, a_{2} \in A$ are given, such that $\partial_{\mathscr{P}}\left(a_{1}\right)=\partial_{\mathscr{P} \prime}\left(a_{2}\right)$. Then:

1. There exist elements $a_{3}, \ldots, a_{s}, \ldots \in A$ satisfying

$$
\partial_{\mathscr{P}}\left(a_{s}\right)=\partial_{\mathscr{P} \prime}\left(a_{s+1}\right) .
$$

2. All elements $a_{1}, \ldots, a_{s}, \ldots$ are in involution with respect to both Poisson structures, i.e.,

$$
\left\{a_{\alpha}, a_{\beta}\right\}_{\mathscr{P}}=\left\{a_{\alpha}, a_{\beta}\right\}_{\mathscr{P} \prime}=0
$$

for all $\alpha, \beta \geq 1$. 


\section{The Nijenhuis bracket}

Consider a form-valued derivation $\Omega \in D_{1}\left(\Lambda^{k}\right)$ and the Lie derivative

$$
\mathrm{L}_{\Omega}=\left[d, \mathrm{i}_{\Omega}\right]: \Lambda^{j} \rightarrow \Lambda^{k+j}
$$

where $\mathrm{i}_{\Omega}$ is defined by the composition

$$
D_{1}\left(\Lambda^{k}\right) \otimes_{A} \Lambda^{j} \stackrel{\mathrm{i}}{\longrightarrow} \Lambda^{k} \otimes_{A} \Lambda^{j-1} \stackrel{\wedge}{\longrightarrow} \Lambda^{k+j-1}
$$

Proposition 8 The above Lie derivative possesses the following properties:

1. $\mathrm{L}_{\Omega}\left(\omega \wedge \omega^{\prime}\right)=\mathrm{L}_{\Omega}(\omega) \wedge \omega^{\prime}+(-1)^{k j} \omega \wedge \mathrm{L}_{\Omega}\left(\omega^{\prime}\right)$,

2. $\left[\mathrm{L}_{\Omega}, d\right]=0$,

3. $\mathrm{L}_{\omega \wedge \Omega}=\omega \wedge \mathrm{L}_{\Omega}+(-1)^{k+j} d \omega \wedge \mathrm{i}_{\Omega}$.

Here $\omega \in \Lambda^{j}, \omega^{\prime} \in \Lambda^{j^{\prime}}, \Omega \in D_{1}\left(\Lambda^{k}\right)$.

Other basic properties of the Nijenhuis bracket are presented in the following

Proposition 9 Let $\Omega \in D_{1}\left(\Lambda^{j}\right), \Omega^{\prime} \in D_{1}\left(\Lambda^{j^{\prime}}\right), \Omega^{\prime \prime} \in D_{1}\left(\Lambda^{j^{\prime \prime}}\right)$, and $\omega \in \Lambda^{i}$. Then:

1. $\left[\left[\Omega, \Omega^{\prime}\right]\right]^{\mathrm{n}}+(-1)^{j j^{\prime}}\left[\left[\Omega^{\prime}, \Omega\right]\right]^{\mathrm{n}}=0$,

2. $\left[\left[\Omega,\left[\left[\Omega^{\prime}, \Omega^{\prime \prime}\right]\right]^{\mathrm{n}}\right]\right]^{\mathrm{n}}=\left[\left[\left[\left[\Omega, \Omega^{\prime}\right]\right]^{\mathrm{n}}, \Omega^{\prime \prime}\right]^{\mathrm{n}}+(-1)^{j j^{\prime}}\left[\left[\Omega^{\prime},\left[\left[\Omega, \Omega^{\prime \prime}\right]\right]^{\mathrm{n}}\right]\right]^{\mathrm{n}}\right.$,

3. $\left[\left[\Omega, \omega \wedge \Omega^{\prime}\right]\right]^{\mathrm{n}}=\mathrm{L}_{\Omega}(\omega) \wedge \Omega^{\prime}+(-1)^{i j} \omega \wedge\left[\left[\Omega, \Omega^{\prime}\right]\right]^{\mathrm{n}}-(-1)^{(j+1)\left(i+j^{\prime}\right)} d \omega \wedge \mathrm{i}_{\Omega^{\prime}}(\Omega)$,

4. $\left.\left[\mathrm{L}_{\Omega}, \mathrm{i}_{\Omega^{\prime}}\right]=(-1)^{j} \mathrm{~L}_{\mathrm{i}_{\Omega^{\prime}}} \Omega+\mathrm{i}_{\left[\Omega, \Omega^{\prime}\right.}\right]^{\mathrm{n}}$,

5. $\mathrm{i}_{\Omega}\left[\left[\Omega^{\prime}, \Omega^{\prime \prime}\right]\right]^{\mathrm{n}}=\left[\left[\mathrm{i}_{\Omega} \Omega^{\prime}, \Omega^{\prime \prime}\right]^{\mathrm{n}}+(-1)^{(j+1) j^{\prime}}\left[\left[\Omega^{\prime}, \mathrm{i}_{\Omega} \Omega^{\prime \prime}\right]\right]^{\mathrm{n}}+(-1)^{j^{\prime}} \mathrm{i}_{\left[\Omega, \Omega^{\prime}\right.}\right]^{\mathrm{n}} \Omega^{\prime \prime}$ $-(-1)^{\left(j^{\prime}+1\right) j^{\prime \prime}} \mathrm{i}_{\left[\Omega, \Omega^{\prime \prime}\right]^{\mathrm{n}}} \Omega^{\prime}$.

On decomposable elements the Nijenhuis bracket acts as follows. Let $\omega \in \Lambda^{j}, \omega^{\prime} \in \Lambda^{j^{\prime}}$, $X, X^{\prime} \in D_{1}(A)$. Then

$$
\begin{aligned}
{\left[\left[\omega \wedge X, \omega^{\prime} \wedge X^{\prime}\right]\right]^{\mathrm{n}} } & =\omega \wedge \omega^{\prime} \wedge\left[X, X^{\prime}\right]+\mathrm{L}_{\omega \wedge X}\left(\omega^{\prime}\right) \wedge X^{\prime}-(-1)^{j j^{\prime}} \mathrm{L}_{\omega^{\prime} \wedge X^{\prime}}(\omega) \wedge X \\
& =\omega \wedge \omega^{\prime} \wedge\left[X, X^{\prime}\right]+\omega \wedge \mathrm{L}_{X}\left(\omega^{\prime}\right) \wedge X^{\prime}-\mathrm{L}_{X^{\prime}}(\omega) \wedge \omega^{\prime} \wedge X \\
& +(-1)^{j} d \omega \wedge \mathrm{i}_{X}\left(\omega^{\prime}\right) \wedge X^{\prime}+(-1)^{j} \mathrm{i}_{X^{\prime}}(\omega) \wedge d \omega^{\prime} \wedge X .
\end{aligned}
$$

A derivation $\mathscr{N} \in D_{1}\left(\Lambda^{1}\right)$ is called integrable if

$$
[[\mathscr{N}, \mathscr{N}]]^{\mathrm{n}}=0
$$

With any integrable derivation one can associate a complex

$$
0 \longrightarrow D_{1}(B) \stackrel{\partial_{\mathscr{N}}}{\longrightarrow} \cdots \longrightarrow D_{1}\left(\Lambda^{j}(B)\right) \stackrel{\partial_{\mathscr{N}}}{\longrightarrow} D_{1}\left(\Lambda^{j+1}(B)\right) \longrightarrow \cdots
$$

where $\partial_{\mathscr{N}}=[[\mathscr{N}, \cdot]]^{\mathrm{n}}$.

Such structures, in particular, arise in an algebraic model of flat connections. 


\section{Flat connections}

Let $A$ and $B$ be $\mathbb{k}$-algebras and $\gamma: A \rightarrow B$ be a homomorphism. Then $B$ is an $A$-algebra and one can consider the module $D_{1}(A, B)$ of $B$-valued derivations $A \rightarrow B$. For any $X \in D_{1}(B)$ denote by $\left.X\right|_{A} \in D_{1}(A, B)$ its restriction to $A$.

A connection is a $B$-homomorphism $\nabla: D_{1}(A, B) \rightarrow D_{1}(B)$ such that $\left.\nabla(X)\right|_{A}=X$. A vector-valued form $U_{\nabla} \in D_{1}(B)$ defined by

$$
\mathrm{i}_{X}\left(U_{\nabla}\right)=X-\nabla\left(\left.X\right|_{A}\right), \quad X \in D_{1}(B),
$$

is called the connection form. For any two derivations $X, X^{\prime} \in D_{1}(A, B)$ we set

$$
R_{\nabla}\left(X, X^{\prime}\right)=\left[\nabla(X), \nabla\left(X^{\prime}\right)\right]-\nabla\left(\nabla(X) \circ X^{\prime}-\nabla\left(X^{\prime}\right) \circ X\right) ;
$$

$R_{\nabla}$ is the curvature of $\nabla$. A connection is flat if $R_{\nabla}=0$.

9.1 Nijenhuis cohomologies associated to a connection

Theorem 3 Let $\nabla$ be a connection. Then

$$
\mathrm{i}_{X}\left(\mathrm{i}_{X^{\prime}}\left(\left[\left[U_{\nabla}, U_{\nabla}\right]\right]^{\mathrm{n}}\right)\right)=2 R_{\nabla}\left(\left.X\right|_{A},\left.X^{\prime}\right|_{A}\right)
$$

for any $X, X^{\prime} \in D_{1}(B)$.

Hence, to any flat connection, i.e., to a connection whose curvature vanishes, we associate a Nijenhuis complex with $\mathscr{N}=U_{\nabla}$. In applications, its vertical subcomplex is useful:

$$
0 \longrightarrow D_{1}^{v}(B) \stackrel{\partial_{\mathscr{N}}}{\longrightarrow} \cdots \longrightarrow D_{1}^{v}\left(\Lambda^{j}(B)\right) \stackrel{\partial_{\mathscr{N}}}{\longrightarrow} D_{1}^{v}\left(\Lambda^{j+1}(B)\right) \longrightarrow \cdots
$$

where $D_{1}^{v}(P)=\left\{X \in D_{1}(P)|X|_{A}=0\right\}$. Denote its cohomologies by $H^{j}(B, \nabla)$.

9.2 Nijenhuis cohomologies: $H^{0}, H^{1}$, and $H^{2}$

Theorem 4 Let $\nabla$ be a flat connection. Then:

1. The cohomology groups $H^{j}(B, \nabla)$ inherit the inner product operation,

$$
\mathrm{i}: H^{j}(B, \nabla) \times H^{j^{\prime}}(B, \nabla) \rightarrow H^{j+j^{\prime}-1}(B, \nabla) \text {. }
$$

In particular, the group $H^{1}(B, \nabla)$ is an associative algebra represented in endomorphisms of $H^{0}(B, \nabla)$ :

$$
\begin{aligned}
& \text { i: } H^{1}(B, \nabla) \times H^{1}(B, \nabla) \rightarrow H^{1}(B, \nabla), \\
& \text { i: } H^{1}(B, \nabla) \times H^{0}(B, \nabla) \rightarrow H^{0}(B, \nabla) .
\end{aligned}
$$

2. The cohomology groups $H^{j}(B, \nabla)$ inherit the Nijenhuis bracket,

$$
[\cdot \cdot \cdot \cdot]^{\mathrm{n}}: H^{j}(B, \nabla) \times H^{j^{\prime}}(B, \nabla) \rightarrow H^{j+j^{\prime}}(B, \nabla) .
$$

In particular, $H^{0}(B, \nabla)$ is a Lie algebra:

$$
[[\cdot, \cdot]]^{\mathrm{n}}: H^{0}(B, \nabla) \times H^{0}(B, \nabla) \rightarrow H^{0}(B, \nabla) .
$$


9.3 Application to differential equations

Let $\mathscr{E} \subset J^{\infty}(\pi)$ be an infinitely prolonged differential equation in the jet bundle of a bundle $\pi: E \rightarrow M$. The bundle $\pi_{\infty}: \mathscr{E} \rightarrow M$ is always endowed with a natural flat connection $\mathscr{C}$ (the Cartan connection, see Refs [1,15]) and taking

$$
\gamma=\pi_{\infty}^{*}: A=C^{\infty}(M) \rightarrow B=C^{\infty}(\mathscr{E})
$$

we obtain the picture considered above.

Let us use the notation $H^{j}(\mathscr{E}, \mathscr{C})$ for the cohomology groups arising in this case.

9.4 The main result

Theorem 5 For any formally integrable equation $\mathscr{E}$ that surjectively projects to $J^{0}(\pi)$ one has:

1. $H^{0}(\mathscr{E}, \mathscr{C})$ coincides with the Lie algebra sym $\mathscr{E}$ of higher symmetries of $\mathscr{E}$.

2. Elements of $H^{1}(\mathscr{E}, \mathscr{C})$ act on sym $\mathscr{E}$ and thus are identified with recursion operators for symmetries.

3. On the other hand, elements of $H^{1}(\mathscr{E}, \mathscr{C})$ can be understood as classes of nontrivial infinitesimal deformations of the equation structure.

4. $H^{2}(\mathscr{E}, \mathscr{C})$ contains obstructions to prolongation of infinitesimal deformations up to formal ones.

9.5 Commutative hierarchies

Let $(B, \nabla)$ be an algebra with flat connection. For $X=X_{0} \in H^{0}(B, \nabla)$ and $R \in H^{1}(B, \nabla)$, use the notation $R(X)=\mathrm{i}_{X}(R), X_{n}=R^{n}(X), n=0,1,2 \ldots$

Theorem 6 Assume that $H^{2}(B, \nabla)=0$. Then for any $X, Y \in H^{0}(B, \nabla)$ and $R \in H^{1}(B, \nabla)$ for all $m, n \in \mathbb{Z}_{+}$one has

$$
\left[X_{m}, Y_{n}\right]=[X, Y]_{m+n}+\sum_{i=0}^{n-1}\left([[X, R]]^{\mathrm{n}}\left(Y_{i}\right)\right)_{m+n-i-1}-\sum_{j=0}^{m-1}\left([[Y, R]]^{\mathrm{n}}\left(X_{j}\right)\right)_{m+n-j-1} .
$$

Corollary 1 If $[[X, R]]^{\mathrm{n}}=\left[[Y, R]^{\mathrm{n}}=0\right.$ and $[X, Y]=0$ then $\left[X_{m}, Y_{n}\right]=0$ for all $m, n \in \mathbb{Z}_{+}$.

Remark 6 If $\mathscr{E}$ is a scalar evolutionary equation of order $>1$ then $H^{2}(\mathscr{E}, \mathscr{C})=0$.

\subsection{Bi-complex}

Let $\mathscr{N} \in D_{1}\left(\Lambda^{1}\right)$ be an integrable element, i.e., $[[\mathscr{N}, \mathscr{N}]]^{\mathrm{n}}=0$. Then the operator

$$
d_{\mathscr{N}}=\mathrm{L}_{\mathscr{N}}: \Lambda^{j} \rightarrow \Lambda^{j+1}
$$

is a differential: $d_{\mathscr{N}} \circ d_{\mathscr{N}}=0$. Moreover, one has

$$
\left[d, d_{\mathscr{N}}\right]=0
$$

and consequently the pair $\left(d_{\mathscr{N}}, \bar{d}_{\mathscr{N}}\right)$, where $\bar{d}_{\mathscr{N}}=d-d_{\mathscr{N}}$, constitutes a bi-complex that converges to the de Rham cohomologies of $B$.

In the case of differential equations $\left(A=C^{\infty}(M), B=C^{\infty}(\mathscr{E})\right.$, and $\mathscr{N}$ is the connection form of the Cartan connection in the bundle $\pi_{\infty}: \mathscr{E}^{\infty} \rightarrow M$ ), this bi-complex coincides with the variational bi-complex, or Vinogradov's $\mathscr{C}$-spectral sequence, see Ref. [24,28]. 


\section{More brackets...}

To conclude, note that several more brackets can be constructed in a similar way.

1. First, mention the Nijenhuis-Richardson bracket

$$
[[\cdot, \cdot]]^{\mathrm{r}}: D_{1}\left(\Lambda^{j}\right) \otimes_{A} D_{1}\left(\Lambda^{j^{\prime}}\right) \rightarrow D_{1}\left(\Lambda^{j+j^{\prime}-1}\right)
$$

that can be defined by

$$
\left.\left[\mathrm{i}_{\Omega}, \mathrm{i}_{\Omega^{\prime}}\right]=\mathrm{i}_{\left[\Omega, \Omega^{\prime}\right.}\right]^{\mathrm{r}}
$$

and is of the form

$$
\left[\left[\Omega, \Omega^{\prime}\right]^{\mathrm{r}}=\mathrm{i}_{\Omega}\left(\Omega^{\prime}\right)-(-1)^{(i-1)(j-1)} \mathrm{i}_{\Omega^{\prime}}(\Omega)\right.
$$

and is one of the classical and well known brackets.

Two more brackets arise also if we fix a Poisson structure $\mathscr{P} \in D_{2}(A)$ or a Nijenhuis structure $\mathscr{N} \in D_{1}(\Lambda)$ :

2. Consider the inner product

$$
\text { i : } D_{i}\left(\Lambda^{1}\right) \otimes_{A} D_{k}(A) \rightarrow D_{i+k-1}(A) \text {. }
$$

Then for $\Omega \in D_{i}\left(\Lambda^{1}\right)$ the following "Lie derivative" arises:

$$
\mathrm{L}_{\Omega}^{\mathscr{P}}=\left[\partial_{\mathscr{P}}, \mathrm{i}_{\Omega}\right]: D_{k}(A) \rightarrow D_{k+i}(A)
$$

and one can introduce a bracket

$$
[[\cdot, \cdot]]^{\mathscr{P}}: D_{i}\left(\Lambda^{1}\right) \times D_{i^{\prime}}\left(\Lambda^{1}\right) \rightarrow D_{i+i^{\prime}}\left(\Lambda^{1}\right)
$$

by

$$
\mathrm{L}_{\left[\Omega, \Omega^{\prime}\right]^{\mathscr{P}}}^{\mathscr{P}}=\left[\mathrm{L}_{\Omega}^{\mathscr{P}}, \mathrm{L}_{\Omega^{\prime}}^{\mathscr{P}}\right]
$$

3. In a similar way, one can consider the inner product

$$
\text { i: } D_{1}\left(\Lambda^{i}\right) \otimes_{A} D_{1}\left(\Lambda^{k}\right) \rightarrow D_{1}\left(\Lambda^{i+k-1}\right)
$$

and the "Lie derivative"

$$
\mathrm{L}_{\Omega}^{\mathscr{N}}=\left[\partial_{\mathscr{N}}, \mathrm{i}_{\Omega}\right]: D_{1}\left(\Lambda^{k}\right) \rightarrow D_{1}\left(\Lambda^{k+i}\right)
$$

$\Omega \in D_{1}\left(\Lambda^{i}\right)$. Then a new bracket

$$
[[\cdot, \cdot]]^{\mathscr{N}}: D_{1}\left(\Lambda^{i}\right) \times D_{1}\left(\Lambda^{i^{\prime}}\right) \rightarrow D_{1}\left(\Lambda^{i+i^{\prime}}\right)
$$

is defined by

$$
\mathrm{L}_{\left.\left[\Omega, \Omega^{\prime}\right]\right]^{\mathscr{N}}}^{\mathscr{N}}=\left[\mathrm{L}_{\Omega}^{\mathscr{N}}, \mathrm{L}_{\Omega}^{\mathscr{N}}\right]
$$




\section{$11 \ldots$ and when brackets fail to arise}

One can also define the inner products

$$
\mathrm{i}: D_{i}\left(\Lambda^{j}\right) \otimes_{A} \Lambda^{k} \rightarrow \Lambda^{k+j-i}
$$

and

$$
\text { i : } D_{i}\left(\Lambda^{j}\right) \otimes_{A} D_{k}(A) \rightarrow D_{k-j+i}(A)
$$

together with the corresponding Lie actions

$$
\mathrm{L}_{\Omega}=\left[d, \mathrm{i}_{\Omega}\right]: \Lambda^{k} \rightarrow \Lambda^{k+j-i+1}
$$

and

$$
\mathrm{L}_{\Omega}^{\mathscr{P}}=\left[\partial_{\mathscr{P}}, \mathrm{i}_{\Omega}\right]: D_{k}(A) \rightarrow D_{k-j+i+1}(A),
$$

where $\Omega \in D_{i}\left(\Lambda^{j}\right)$. Of course, it is tempting to find the elements $\left[\left[\Omega, \Omega^{\prime}\right]\right]$ and $\left[\left[\Omega, \Omega^{\prime}\right]^{\mathscr{P}}\right.$ such that

$$
\mathrm{L}_{\left.\llbracket \Omega, \Omega^{\prime}\right]}=\left[\mathrm{L}_{\Omega}, \mathrm{L}_{\Omega^{\prime}}\right], \quad \mathrm{L}_{\llbracket \Omega, \Omega^{\prime} \rrbracket}^{\mathscr{P}}=\left[\mathrm{L}_{\Omega}^{\mathscr{P}}, \mathrm{L}_{\Omega^{\prime}}^{\mathscr{P}}\right],
$$

but in general such elements do not exist (see discussion of these matters in Ref. [26]).

\section{References}

1. Bocharov, A. V., Chetverikov, V. N., Duzhin, S. V., et al., Symmetries and Conservation Laws for Differential Equations of Mathematical Physics, xiv+333 pp. Amer. Math. Soc., Providence, RI (1999). Edited and with a preface by I. Krasil' shchik and A. Vinogradov.

2. J.-L. Brylinski, A differential complex for Poisson manifolds. Source, J. Differential Geom. 28, (1988), no. $1,93-114$

3. A. Frölicher, A. Nijenhuis, Theory of vector valued differential forms. Part I., Indagationes Math., 18 (1956), 338-360.

4. A. Frölicher, A. Nijenhuis, Invariance of vector form operations under mappings, Comm. Math. Helv, 34 (1960), 227-248.

5. Kersten P., Krasil' shchik I., Verbovetsky A., Hamiltonian operators and $\ell^{*}$-coverings, J. Geom. and Phys. 50, pp. 273-302 (2004), arXiv:math.DG/0304245

6. P. Kersten, I. Krasil'shchik, A. Verbovetsky, R. Vitolo, this issue.

7. I. Krasil'shchik, Algebras with flat connections and symmetries of differential equations, in: Lie Groups and Lie Algebras: Their Representations, Generalizations and Applications, Kluwer Acad. Publ., Dordrecht/Boston/London, 1998, 407-424.

8. I. Krasil'shchik, Schouten brackets and canonical algebras, Global Anal. and Math. Physics, Voronezh State University, 1987. English translation in Springer Lecture Notes in Math., 1334, Springer-Verlag, 1988.

9. I. Krasil'shchik, Some new cohomological invariants of nonlinear differential equations, Differential Geometry and Its Appl., 2, (1992), no. 4, 307-350.

10. I. Krasil' shchik, Supercanonical algebras and Schouten brackets, Mat. Zametki, 49 (1991), no. 1. English translation in Soviet Mat. Zametki, 70-76.

11. I. Krasil'shchik, Characteristics of linear differential operators over commutative algebras, Acta Appl. Math., 49, (1997), no. 3, 257-269.

12. I. Krasil'shchik, Calculus over commutative algebras: a concise user guide, Acta Appl. Math., Volume 49, Issue 3, December 1997, 235-248.

13. I. Krasil's shchik, Hamiltonian cohomologies of canonical algebras, Dokl. AN SSSR, 251, (1980), no. 6 (in Russian, English translation in: Soviet Math. Dokl.)

14. I. Krasil's shchik, Poincare $\delta$-lemma for smooth algebras, Acta Appl. Math., 49, (1997), no. 3, $249-255$.

15. I. Krasil'shchik, V. Lychagin, A. Vinogradov, Geometry of Jet Spaces and Nonlinear Differential Equations, Advanced Studies in Contemporary Mathematics, 1 (1986), Gordon and Breach, New York, London. $\mathrm{xx}+441 \mathrm{pp}$.

16. V. Lychagin, Color calculus and color quantizations, Acta Appl. Math., 41 (1995), 193-226 
17. A. Lichnerowicz, Les variétés de Poisson et leurs algèbres de Lie associées, J. Diff. Geom. 12 (1977), 253-300.

18. F. Magri, A simple model of the integrable Hamiltonian equation, J. Math. Phys., 19 (1978), 1156-62.

19. A. Nijenhuis, Jacobi-type identities for bilinear differential concomitants of certain tensor fields I, Indagationes Math., 17 (1955) 390-403.

20. A. Nijenhuis, R. Richardson, Cohomology and deformations in graded Lie algebras, Bull. Amer. Math. Soc., 72 (1966), 1-29.

21. A. Nijenhuis, R. Richardson, Deformation of Lie algebra structures, J. Math. Mech., 17 (1967), 89-105.

22. J. A. Schouten, Über Differentialkonkomitanten zweier kontravarianten Grössen, Indag. Math., 2 (1940), 449-452.

23. J. A. Schouten, On the differential operators of the first order in tensor calculus, In: Convegno Int. Geom. Diff. Italia, 1953, Edizione Cremonese, Roma, 1-7.

24. Vinogradov A. M., Cohomological Analysis of Partial Differential Equations and Secondary Calculus, vi+247 pp. Amer. Math. Soc., Providence, RI (2001).

25. A. Vinogradov, The logic algebra for the theory of linear differential operators, Soviet Math. Dokl., 13 (1972), 1058-1062.

26. A. Vinogradov, The union of the Schouten and Nijenhuis brackets, cohomology, and superdifferential operators, Mat. Zametki, 47 (1990), no. 6, 138-140. English translation in Russian Math. Notes.

27. A. Vinogradov, I. Krasil'shchik, What is the Hamiltonian formalism? London Math. Soc. Lect. Notes Ser., 60 (1981), Cambridge Univ. Press, London, 241-266.

28. A. Vinogradov, The $\mathscr{C}$-spectral sequence, Lagrangian formalism, and conservation laws I, II, J. Math. Anal. Appl., 100 (1984), 1-129. 